YITP-08-32

\title{
Chiral Symmetry and Heavy-Ion Collisions
}

\author{
Kenji Fukushima \\ Yukawa Institute for Theoretical Physics, \\ Kyoto University, Kyoto 606-8502, Japan
}

\begin{abstract}
I revisit the phase structure of hot and dense matter out of quarks and gluons with some historical consideration on the color deconfinement and chiral phase transitions. My goal is to make clear which part of the QCD phase diagram is under theoretical control and which part is not. I demonstrate that an uncommon but logically possible scenario other than the standard phase diagram cannot be ruled out. My emphasis is on the concern that one should correctly understand what kind of phenomenon occurs associated with the phase boundary line on the diagram. It is not quite obvious, in particular, where chiral symmetry restoration plays a phenomenological role in the temperature and baryon density plane except at the QCD (chiral) critical point.
\end{abstract}

\section{How is the QCD phase diagram built?}

The quest for a new state of matter out of quarks and gluons is becoming a matured subject in physics founded on Quantum Chromodynamics (QCD). We are opening an epoch of exact science for a quark-gluon plasma. There have already come convincing experimental data from RHIC at the Brookhaven National Laboratory and more will be forthcoming from LHC at CERN in a couple of years. So far, the intrinsic properties of transient matter are mainly concerned from the experimental point of view, but an enough deal of compilation of experimental outputs would enable us to access the detailed structure of the QCD phase transitions directly.

In fact, it is the conjectured QCD phase diagram that has been a guiding principle leading to feasible research projects to create hot and dense QCD matter in laboratory. Since the standard shape of the QCD phase diagram is widely prevailing as a common knowledge, it is more or less difficult for us to doubt such an almost unanimous assent. If one tries to figure it out somehow from theory, however, one should realize that our current knowledge does not suffice to guarantee that the standard scenario is a unique possibility. To understand this it would be instructive to start with a historical overview of how the QCD phase diagram is built.

There is a consensus in the community that the very first QCD phase diagram with the temperature $T$ and the baryon density $n_{\mathrm{B}}$ axes appeared in a paper by Cabibbo and Parisi in 1975 [1. They interpreted the exponentially increasing spectrum by Hagedorn (that is, the Hagedorn spectrum) as "deconfinement" and insisted that quarks are confined in the low- $T$ and low- $n_{\mathrm{B}}$ region and deconfined otherwise. The "quarter sector" shape of the phase boundary line, which is quite familiar to us today, first revealed itself then. I would like to remind that chiral symmetry plays no role at all in this context. Of course, the theory of chiral symmetry restoration was developing almost parallelly but separately from the color deconfinement theory. 


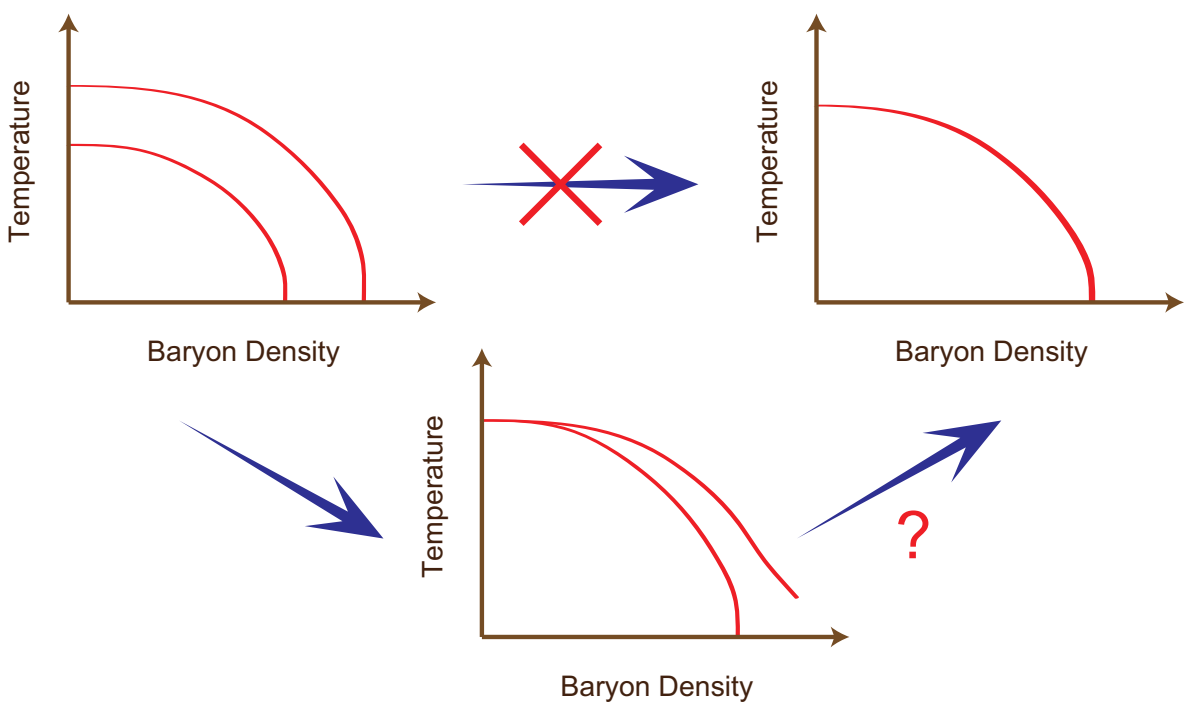

Figure 1. Schematic evolution of the possible phase diagrams in the temperature $T$ and baryon density $n_{\mathrm{B}}$ (or chemical potential $\mu$ ) plane. Two red curves represent the deconfinement and chiral restoration phase boundaries. For simplicity the mixed-phase region associated with the first-order transition is not considered or the horizontal axis should be not $n_{\mathrm{B}}$ but $\mu$, which was not taken quite seriously in the 80 's.

Then, a dramatic leap, in my opinion, was made in 1983 into a prototype of what we now know as the QCD phase diagram. Let me select out two historic figures depicted both by Baym. One is found in his contribution to the Bielefeld proceedings published in 1982 [2]. It might look bizarre to draw two distinct boundary lines on the phase diagram like shown in the upper-left of Fig. 1 which is rather exotic to us but was a serious candidate in 1982. Each boundary line represents either deconfinement or chiral symmetry restoration. Another important and more famous figure is put in the NSAC Long Range Plan for Nuclear Science in 1983 which is readily available also from a nice review article on the RHIC history [3. This figure had undergone an important evolution from a double-boundary phase diagram to a single-boundary shape like sketched in the upper-right of Fig. 1.

What caused such a qualitative difference between 1982 and 1983? The answer lies in a situational change in the lattice Monte-Carlo simulation by the Illinois group published in 1983 [4]. It was claimed that deconfinement and chiral symmetry restoration take place at almost the same temperature in both $\mathrm{SU}(2)$ and $\mathrm{SU}(3)$ gauge theories. The locking of two transitions or crossovers has been confirmed by more accurate simulations, which makes most people in the community believe in the single-boundary phase diagram. [Whether the locking is complete or approximate is still under dispute [5, 6, but in any case two critical temperatures are close to each other.]

This deduction, however, skips one logical step, I would say. Our knowledge from the Monte-Carlo simulation is limited to the region where the baryon density is much smaller than the temperature. Therefore, the single-boundary diagram is too severely constrained than what we can conclude from the lattice results. The logical deduction 
from the upper-left of Fig. 1 and the coincidence of two critical temperatures at zero density leads to a phase diagram as given in the lower-middle of Fig. 1. I would dare not deny the conventional phase diagram, however, by this argument; in Fig. 1 the upper-right is still a possible candidate as a special reduction from the lower-middle. My point is that we have not discovered any evidence for such a reduction.

We shall assume a double-boundary phase diagram for the moment. Then, we would hit on the following question; which line is which of deconfinement and chiral symmetry restoration? Unfortunately no theoretical argument can tell it in a rigorous way. Some discussions [7, 8, imply that quark confinement should be accompanied by the spontaneous breaking of chiral symmetry in the vacuum, but these theoretical arguments do not hold in a medium. I will present some model analyses on what will transpire later.

Here let us turn to another big stream toward the QCD phase diagram. That is, a pile of achievements based on the low-energy chiral effective model. The most prominent finding from model analyses is, as I will explain below, the critical end-point that is sometimes referred to as the QCD critical point. Since I cannot enumerate all the chiral effective models such as the linear and non-linear sigma models, the Nambu-Jona-Lasinio (NJL) model, the chiral random matrix model, the instanton liquid model, and so on, I will focus only on the NJL model in this article. This is because the QCD critical point was first pointed out in a work based on the NJL model [9] and also because my discussions will be followed later by state-of-the-art results in a closely related model with the Polyakov loop incorporated.

One of the widely-known early works by means of the NJL model is that done by Hatsuda and Kunihiro [10. They drew a line on the $T$ and $\mu$ plane in 1985 that separates the chiral symmetric and broken phases with an explicit statement that they neglected any effect of confinement. Note that the NJL model at finite $T$ is premised on deconfined matter in terms of quarks. In 1986 Asakawa and Yazaki got aware of the first-order phase transition at high density. That means that the chiral phase transition is continuous in the small- $\mu$ region and discontinuous in the large- $\mu$ region. There must be a resultant terminal point where the phase transition ceases to be of first-order. This end-point of the first-order boundary is commonly called the chiral critical end-point (CEP) or simply the QCD critical point. I think that the former makes more sense though the latter seems more prevailing. I would emphasize that the so-called QCD critical point has an origin in chiral dynamics alone and no direct link to confinement and deconfinement.

In fact, it is extremely important to keep in mind the fact that the QCD critical point is associated with chiral symmetry. For instance, our limited knowledge cannot exclude the following scenario however unlikely (and undesirable) it sounds. The density effect, as I mentioned, could make the deconfinement phase transition take place in a totally different way from chiral restoration. If the QCD critical point sitting on the chiral transition line is located far away from deconfinement which predominantly determines the equation of state, it should be hardly conceivable to expect any significant influence on the hydrodynamic path even near the QCD critical point. This is still logically plausible.

We have looked over theoretical understanding and remaining possibilities of the QCD phase diagram on a general footing. We shall now proceed into concrete studies using the effective model in the subsequent sections. 


\section{Is it deconfinement or chiral restoration?}

In general there should be a physical quantity which behaves differently from one phase to the other to define the phase transition. It is the chiral condensate $\langle\bar{q} q\rangle$ in the case of the chiral symmetry breaking and the Polyakov loop $\ell$ serves as an order parameter for deconfinement. That is,

$$
\begin{aligned}
& \text { Chiral Symmetric Phase } \rightarrow\langle\bar{q} q\rangle=0, \\
& \text { Chiral Broken Phase } \rightarrow\langle\bar{q} q\rangle \neq 0, \\
& \text { Quark Confinement Phase } \rightarrow \ell=0, \\
& \text { Quark Deconfinement Phase } \rightarrow \ell \neq 0 .
\end{aligned}
$$

Theoretically speaking, however, $\langle\bar{q} q\rangle$ could be exactly zero only when chiral symmetry is exact, i.e., the quark mass $m_{q}$ is strictly zero. As for deconfinement, similarly, $\ell$ could be zero only when $m_{q}$ is infinitely large (that is, center symmetry is exact in the quenched limit). Therefore none of the above can define an exact critical point in the real world with finite quark masses. Nevertheless, it is still sensible to locate the pseudo-critical point by the peak position of the susceptibility. Remember that the susceptibility with respect to order parameter diverges at the second-order critical point, and thus, it may well have a maximum even in the case of smooth crossover.

Because we have two independent order parameters, it would be rather natural to anticipate two distinct pseudo-critical temperatures. The fact is, however, that the lattice QCD simulations result in the coincidence of two peak positions of the chiral and Polyakov loop susceptibility. It is quite hard to explain this coincidence unless two order parameters are mixed up to form one order parameter [11. I would stress that the mixing argument is not adequate to exclude two peaks in respective susceptibility [12, 13.

Here I will introduce one simple idea that turns out to be quite successful to reproduce simultaneous crossover in terms of $\langle\bar{q} q\rangle$ and $\ell$ as a function of $T$. A textbook knowledge on statistical mechanics reads the partition function for free quarks as $Z_{f}=2 \int d p\left\{\log \left[1+e^{-(E-\mu) / T}\right]^{3}+\log \left[1+e^{-(E+\mu) / T}\right]^{3}\right\}$ with 2 and 3 being spin and color factors, which is modified by the presence of the Polyakov loop into a form of [14, 15, 16]

$$
\begin{aligned}
Z_{f}= & 2 \int d p\left\{\log \left[1+3 \ell e^{-(E-\mu) / T}+3 \bar{\ell} e^{-2(E-\mu) / T}+e^{-3(E-\mu) / T}\right]\right. \\
& \left.+\log \left[1+3 \bar{\ell} e^{-(E+\mu) / T}+3 \ell e^{-2(E+\mu) / T}+e^{-3(E+\mu) / T}\right]\right\}
\end{aligned}
$$

where $e^{-(E-\mu) / T}$ represents one-quark excitation which couples $\ell$ and $e^{-2(E-\mu) / T}$ represents two-quark excitation which has the same color structure as one-anti-quark excitation and thus couples $\bar{\ell}$. The last term, $e^{-3(E-\mu) / T}$, represents the colorless baryon-like excitation. The second line corresponds to the anti-quark contribution. Because the energy dispersion relation $E$ depends on the chiral condensate $\langle\bar{q} q\rangle$ through the constituent quark mass, the coupling between $\ell$ and $\langle\bar{q} q\rangle$ weakens when chiral symmetry is heavily broken. Conversely, as long as $\ell$ is vanishingly small, only the baryon-like excitation is allowed and the chiral phase transition is delayed accordingly. These dynamical entanglement leads to simultaneous crossover of deconfinement and chiral restoration.

We see immediately that the nature of confinement is unchanged for any $\mu$ as long as $T$ is sufficiently small. This is because only the term $e^{-3(E-\mu) / T}$ becomes 

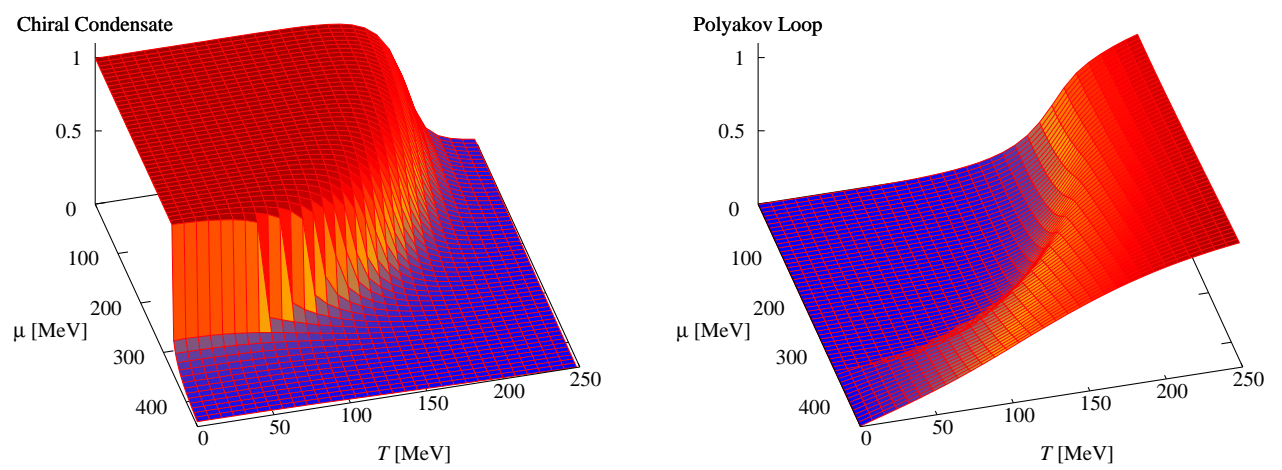

Figure 2. Behavior of order parameters in the $T$ - $\mu$ plane in the PNJL model taken from Ref. [16. Left) Chiral condensate for light (up and down) quarks normalized by the value at $T=\mu=0$. Right) Polyakov loop $\ell$.

dominant at $\mu>E$ and $T \simeq 0$, and all the coupling terms to $\ell$ drop off. The physical interpretation for this is transparent in fact. Let us imagine that we have free quark matter at a certain value of $\mu$. Then, three degenerate quarks with different colors should occupy each level in the phase space up to the Fermi surface and they form a color singlet in momentum space. It does not have to be a spatially compact object like a nucleon. Hence, quark deconfinement in configuration space would make no further qualitative change to degenerate quark matter if seen in momentum space.

As a result of persisting confinement at low $\mu$ the upper and lower lines in the lower-middle of Fig. [1 should correspond to deconfinement and chiral restoration, respectively, according to this intuitive argument. That is actually the case in the model study [16.

\section{What can theoretical models tell?}

In the rest of this article I will address the QCD phase diagram inferred from the recent analyses in the three-flavor NJL model with the Polyakov loop augmented (PNJL model). We see the behavior of the light-quark chiral condensate $\langle\bar{u} u\rangle$ (normalized by the vacuum value) and the Polyakov loop $\ell$ in the $T$ - $\mu$ plane in Fig. 2. Here it should be reiterated that the model parameters are fixed to reproduce the vacuum property and the simultaneous crossover at $T \neq 0$ and $\mu=0$. All the data at $\mu \neq 0$ are thus the model prediction that is to be compared to the lattice QCD simulation in the future when the sign problem will be solved. I remark that color superconductivity is not taken into account in the results displayed in Fig. 2 .

It is clear that the chiral condensate and the Polyakov loop are closely related to each other. We might consider at a first glance that the upper-right of Fig. 1, that is, the standard single-boundary phase diagram might be concluded from these two plots in Fig. 2, That is, however, not a right interpretation.

The chiral condensate has a discontinuous jump in the low- $T$ and high- $\mu$ region. We can perceive a tiny jump in the Polyakov loop as well. This is because of mixing between two order parameters. As we understood, however, the Polyakov loop cannot go large at $T \simeq 0$ regardless of the first-order phase transition. That means that the phase transition signifies a change from a chiral broken confined phase to a chiral symmetric confined phase, if we naively regard $\ell=0$ as confinement. Accordingly 


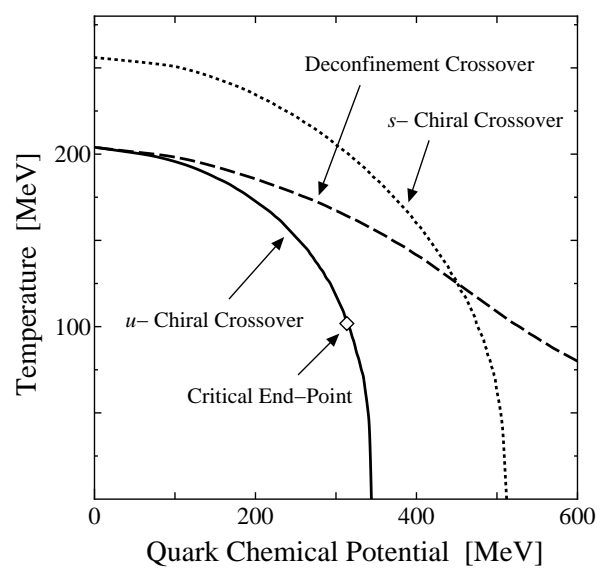

Figure 3. Phase diagram in the three-flavor PNJL model taken from Ref. [16].

the phase structure stemming from this kind of model approaches has no choice but a type of the lower-middle of Fig. 1 .

Indeed, the phase diagram in the three-flavor PNJL model takes an uncommon structure as given in Fig. 3. The boundary lines are determined by the condition that $\langle\bar{u} u\rangle /\langle\bar{u} u\rangle_{0}=0.5$ (solid line) and $\ell=0.5$ (dashed line) with $\langle\bar{u} u\rangle_{0}$ being the chiral condensate in the vacuum. The dotted line represents the chiral crossover associated with strange quarks which spreads over higher temperature due to strange quark mass.

It is remarkable that the locking of deconfinement and chiral restoration no longer persists in the high- $\mu$ region. The physical meaning of the region where $\langle\bar{u} u\rangle \neq 0$ and $\ell \simeq 0$ is not quite straightforward; the system is nearly center symmetric but the relevant degrees of freedom are presumably quarks. Such weird quark matter is named the "quarkyonic phase" by McLerran and Pisarski [17 in the context of the large $N_{c}$ limit. (See also discussions in Ref. 18.)

\section{To what extent can we believe model predictions?}

In the three-flavor PNJL model the QCD critical point is located at $T=102 \mathrm{MeV}$ and $\mu=313 \mathrm{MeV}$. I have to emphasize that this model output (or any other model outputs as well) is nothing more than one suggestive indication and should not be regarded as conclusive.

The low-energy effective model cannot avoid ambiguity involving the model parameter. In a usual prescription the model parameter is fixed at $T=\mu=0$ and assumed to be constant at any $T$ and $\mu$. Hence the thermal excitation of the effective degrees of freedom alone yields the $T$ and $\mu$ dependent contributions. This assumption is maybe acceptable as long as $T$ and $\mu$ are smaller than the model cutoff scale $\Lambda$ which characterizes the relevant energy scale of the effectiveness. This is because the model parameter encompasses the microscopic dynamics of more elementary particles and those particles reside in the energy scale higher than $\Lambda$, so that their excitation effect should be negligible at $T$ and $\mu$ smaller than $\Lambda$.

There are, however, some model parameters that would significantly change the resultant physical predictions. Here I shall briefly address two such examples according 

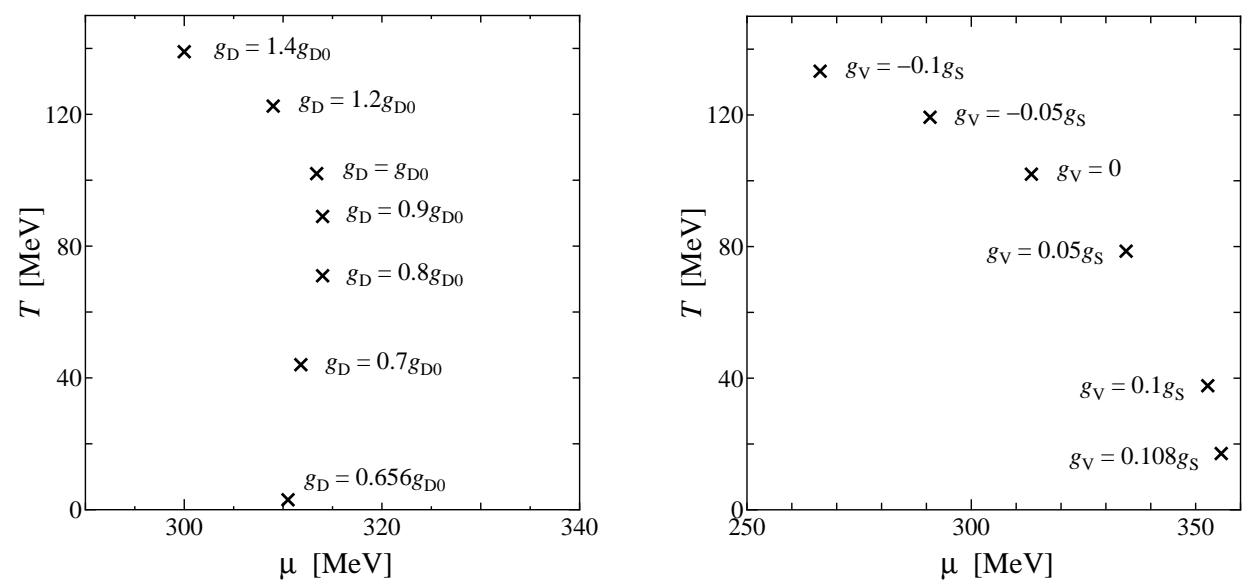

Figure 4. QCD critical point in the PNJL model as a function of the uncontrolled model parameters. Left) 't Hooft interaction dependence. Right) Vector-channel interaction dependence taken from Ref. 16].

to Ref. [16].

The first example is the $\mathrm{U}_{\mathrm{A}}(1)$ symmetry breaking term which is often called the 't Hooft interaction. This term has an origin in the instanton distribution. Because the finite $T$ and $\mu$ effects suppress the instanton excitation, the 't Hooft interaction should diminish exponentially.

The strength of the first-order phase transition strongly depends on the magnitude of the $U_{A}(1)$ symmetry breaking in the case of three-flavor quark matter. Thus, if it gets smaller by the finite- $\mu$ effect, the first-order phase transition region should shrink, and the QCD critical point should move closer to the $T=0$ axis. It will eventually disappear from the phase diagram with the 't Hooft interaction below a certain critical value. The left of Fig. 4 shows the location of the QCD critical point as a function of the $\mathrm{U}_{\mathrm{A}}(1)$ symmetry breaking strength denoted by $g_{\mathrm{D}}$ (and $g_{\mathrm{D} 0}$ being the vacuum value thereof).

It is surprising to see that only $35 \%$ reduction of $g_{\mathrm{D}}$ can be enough for the QCD critical point to disappear. There is no quantitative estimate of how much $g_{\mathrm{D}}$ should be reduced at finite $T$ and $\mu$ (see Ref. [19] for an attempt), but $35 \%$ is within a possible reach as compared to the exponential suppression.

The second example is the strength of the vector-channel interaction. The right of Fig. 4 4 indicates the sensitivity of the QCD critical point as a function of the vectorchannel interaction denoted by $g_{\mathrm{V}}$. Since the zeroth component in the vector channel directly couples the density, the vector-channel interaction could be induced by the finite- $\mu$ environment, which is not quite well known from theory. In the plot of the right of Fig. 4 the interaction strength is given in the unit of the scalar-channel interaction $g_{\mathrm{S}}$. Taking account of the Fierz transformation, $g_{\mathrm{V}}$ could be as large as $g_{\mathrm{S}}$. The critical value, $g_{\mathrm{V}} \simeq 0.11 g_{\mathrm{S}}$, is surprisingly small in this sense.

In view of these above results our conclusion should be that the location of the QCD critical point and even its existence are not under theoretical control and thus not robust. In fact, a recent lattice simulation goes negative against the existence of the QCD critical point [20]. 
Chiral Symmetry and Heavy-Ion Collisions

\section{Remarks}

Finally let me comment on the aspects of chiral symmetry and its dynamics relevant to the heavy-ion collisions.

In general the chiral symmetry breaking and restoration would have an effect mostly on the hadron mass spectrum. The bulk property of hot and dense matter is thus insensitive to chiral dynamics. Consequently the chiral phase transition, if any, cannot bring about a perceivable change in the hydrodynamic evolution. One exception might be the QCD critical point where the equation of state has singularity leading to the focusing effect [21].

Hence, it should be not the bulk thermodynamics but the collective excitations that are more pertinent quantities to probe the chiral properties. The dilepton spectrum and the associated mass shift and/or broadening would be a nice candidate to disclose the chiral characteristics.

One might then think that the dilepton measurement could pinpoint the QCD critical point by the presence of the massless $\sigma$ meson. Unfortunately this idea would not work out because the criticality lies in the space-like channel, while the decay channel is time-like. The spectral function in the space-like region may behave discontinuously from the time-like region [22. Therefore, so far, a promising signature for the QCD critical point is the fluctuation associated with the soft mode in the vicinity of the critical point (see also Ref. 23] for a recent proposal for another signature). As a final remark, I would like to repeat that we should always keep in our mind that the QCD critical point is still a hypothetical idea and its location and existence should be tested by experiments.

This work is in part supported by Yukawa International Program for QuarkHardon Sciences.

[1] N. Cabibbo and G. Parisi, Phys. Lett. B 59, 67 (1975).

[2] G. Baym, in Quark Matter Formation and Heavy Ion Collisions, Proc. Bielefeld Workshop, edited by M. Jacob and H. Satz (World Scientific, Singapore, 1982).

[3] G. Baym, Nucl. Phys. A 698, XXIII (2002) arXiv:hep-ph/0104138.

[4] J. B. Kogut, M. Stone, H. W. Wyld, W. R. Gibbs, J. Shigemitsu, S. H. Shenker and D. K. Sinclair, Phys. Rev. Lett. 50, 393 (1983).

[5] F. Karsch, in this volume of QM proceedings.

[6] Z. Fodor, in this volume of QM proceedings.

[7] A. Casher, Phys. Lett. B 83, 395 (1979).

[8] G. 't Hooft, in Recent Developments in Gauge Theories, edited by G. 't Hooft et al. (Plenum, New York, 1980).

[9] M. Asakawa and K. Yazaki, Nucl. Phys. A 504, 668 (1989).

[10] T. Hatsuda and T. Kunihiro, Phys. Rev. Lett. 55, 158 (1985).

[11] Y. Hatta and K. Fukushima, Phys. Rev. D 69, 097502 (2004) arXiv:hep-ph/0307068.

[12] S. Digal, E. Laermann and H. Satz, Eur. Phys. J. C 18, 583 (2001) arXiv:hep-ph/0007175.

[13] A. Mocsy, F. Sannino and K. Tuominen, Phys. Rev. Lett. 92, 182302 (2004) arXiv:hep-ph/0308135.

[14] K. Fukushima, Phys. Lett. B 591, 277 (2004) arXiv:hep-ph/0310121.

[15] C. Ratti, M. A. Thaler and W. Weise, Phys. Rev. D 73, 014019 (2006) arXiv:hep-ph/0506234.

[16] K. Fukushima, arXiv:0803.3318 [hep-ph].

[17] L. McLerran and R. D. Pisarski, Nucl. Phys. A 796, 83 (2007) arXiv:0706.2191 [hep-ph]].

[18] L. Y. Glozman and R. F. Wagenbrunn, Phys. Rev. D 77, 054027 (2008) arXiv:0709.3080 [hep$\mathrm{ph}]]$.

[19] K. Fukushima, K. Ohnishi and K. Ohta, Phys. Rev. C 63, 045203 (2001) arXiv:nucl-th/0101062.

[20] P. de Forcrand, in this volume of QM proceedings.

[21] C. Nonaka, in this volume of QM proceedings.

[22] H. Fujii, in this volume of QM proceedings.

[23] M. Asakawa, S. A. Bass, B. Muller and C. Nonaka, arXiv:0803.2449 [nucl-th]. 